\title{
Knowledge Modelling for an Electrical PLM System in Aeronautics
}

\author{
MERLO Christophe ${ }^{1,2^{*}}$, VILLENEUVE Éric ${ }^{1}$, BOTTECCHIA Sébastien ${ }^{1}$, \\ DIAZ Pierre ${ }^{1}$ \\ ${ }^{1}$ ESTIA, ESTIA Research, Bidart, France \\ ${ }^{2}$ IMS, UMR5218, Univ. de Bordeaux, France \\ c.merlo@estia.fr
}

\begin{abstract}
Management of the whole product lifecycle is a major stake that most companies have now integrated into their strategies. Research works have been focused on this asset but mainly for mechanical products. In previous work proposes a knowledge modelling approach in a mechanical context, centred on expert users' collaboration. In this paper, we worked with an aeronautical company in charge of electrical maintenance. Its objective was to improve its business processes, from design phase to production phase, by developing a new PLM system, integrated to its electrical CAD software. Our research aim is to evaluate the genericity of previous knowledge modelling approach in this electrical context. We propose then an integrated knowledge model based on three dimensions: (electric) product modelling, (aeronautics retrofitting) process/project modelling and organization/collaboration modelling. We show how this model identifies generic vs expert knowledge and how 'business rules' can be defined to support collaboration and to reduce time of development process. Finally, we propose a global PLM system and we detail the impact on the collaborations and on the whole 'product' lifecycle. This PLM system is actually developed by an electrical CAD editor, ensuring the integration between CAD data and PLM database. We conclude on future work extending this first PLM system: a generic problem-solving approach for capitalizing and reusing knowledge then an augmented reality environment for using PLM product data during installation phase.
\end{abstract}

Keywords: PLM System, Knowledge Modelling, Collaborative Life Cycle Management, Knowledge Reuse 


\section{Introduction}

Manufacturing industries and product development process have strongly evolved for almost twenty years. Many approaches, methods and tools have been implemented to improve the performance of design projects. These approaches and methods can be focused, for example, on multidisciplinary design teams' coordination [1], concurrent engineering [2], or products complexity management [3].

One of the key success factors is the efficiency of collaboration between team members and the relevancy of available design support environments [4]. "To survive in global competitive markets, says [5], the Product Lifecycle Management (PLM) is an emerging philosophy to improve strategic engineering for managing information, processes and resources to support the life cycle of a product".

Actual trends for the industry 4.0 focus on collaborative companies that design, manufacture, produce and deliver complex products and services. Such large companies use generally PLM systems [6] but need to be supported by more relevant knowledge-centred information systems designed for the different phases of the product life-cycle. Therefore, major PLM challenges are based at least on the following key axes [7]:

- an efficient and effective access to the right information, for every stakeholder involved in product development projects, everywhere in the world and when he/she requires this access;

- the definition of an effective information management strategy coupled with a knowledge modelling approach to support the activities of each actor, according to his/her business profile.

In this context, knowledge modelling may be studied through several viewpoints: the modelling of adequate product knowledge, the reengineering of the product development process and the coordination of design-manufacturemaintenance activities as part of the product lifecycle management [8].

By knowledge modelling, it is referred to explicit and implicit knowledge as mentioned in [9] with the aim of structuring knowledge and of proposing a support for team's communication and traceability, through a new generation of PLM system.

In this paper, we study how knowledge modelling helps in defining solutions that will be implemented into a PLM system, allowing the company to become a collaborative company through the whole product lifecycle and to be more competitive. In section 2 we introduce the case study and describe the initial situation of the company and its main process for a specific business activity. Section 3 describes the proposed integrated knowledge model and we show how collaboration between actors is fostered by using the structured knowledge model. Finally, we discuss our proposal and introduce future work that will introduce augmented reality in the production phase of the product life-cycle. 


\section{Case study}

Our case study has been implemented throughout the $2 \pi$-MCO project (PPIMCO: Productivité des PME en Ingénierie électrique pour une Maintenance et une Conception Optimisées / Productivity of SMEs in Electrical Engineering for Optimised Design and Maintenance). This project (2013-2016) was funded by French agency BPI France and the Aquitaine Region, and received Aerospace Valley competitivity pole support. The project leader was an editor of electrical CAD solutions, and the consortium was composed of two "user companies", two research laboratories, two education partners and one "e-learning" company.

\subsection{Airplane retrofit case study}

The $2 \pi$-MCO project aims to implement prototype tools and methods for increasing the productivity of SMEs and Midsize Business companies working in the field of automation and wiring either in maintenance or design (retrofitting). It helps them to remain competitive by reducing design time studies and reducing errors in information transmission.

For that, we focused on industrial processes improvement and capitalizing know-how. First analysis lead to work on:

- Reliable deliverables.

- Greater collaboration between businesses: design, manufacture, installation, testing, documentation, by lower information redundancy; controls automation and document generation; the centralized and shared access to information, etc.

- Implementation of a new PLM environment suitable for this type of business.

The studied company, that we will name "Airplane retrofit" company, is specialist of airplanes maintenance. Its market corresponds to airplanes used for public activities (commercial flights, scientific/public missions or governmental flights). Its main business activities can be divided into two main activities: direct maintenance tasks, or complete airplane retrofitting that implies to redesign internal infrastructures and equipment then install them into the planes. The case study is based on the retrofit activity so we consider the redesigned infrastructure and equipment as the "product" and their installation (assembly) as the production phase.

ESTIA was mandated to support this company to highlight its needs and recommend models, features and software MMI (Man/Machine Interface) proposals, which will be implemented via a new PLM tool. This development will be provided by the software editor, already a supplier of CAD wiring solution. 


\subsection{Airplane retrofit business process}

Our approach relied in this case study on interviews and observations. We met several actors to be able to analyse all phases of the product life-cycle. We aim to define with them what were their activities, what kind of information they need, they produce and they send and finally what tools do they use.

As shown on the diagram below, the product development process of an airplane retrofit can be split into four phases: Customer Specifications, Preliminary Design, Detailed Design and Manufacture before Assembly. A fifth phase that we call the installation/production phase corresponds to all the operations of disassembly of old equipment and assembly of new ones into the airplane.

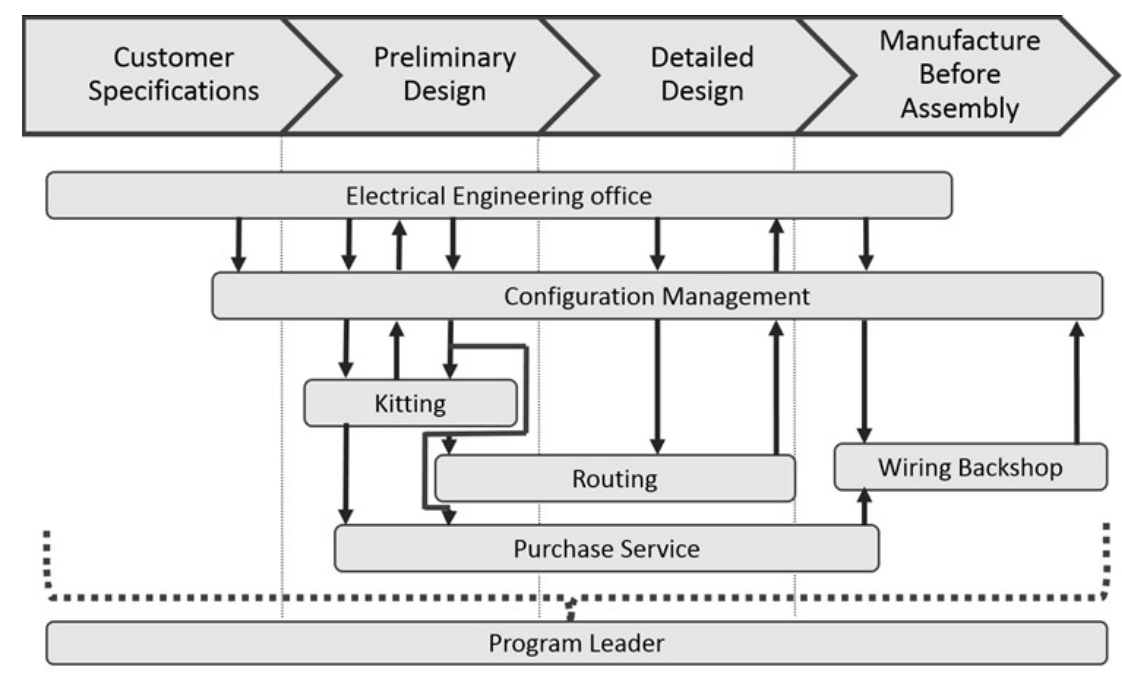

Fig. 1. The studied collaborative product development process

During all phases, several expert actors are involved and need to work together, collaboratively and simultaneously, for a successful outcome of the business agreement with the shortest time. The Program Leader oversees the ongoing project and validates technical design choices with the customer. He is the interface between customer and technical teams. The Electrical Engineering Office performs four types of plans during the entire process to answer to customer specifications, from the most general to the most detailed (Layout Diagram, Block Diagram, Schematic Diagram, Wiring Diagram). Each diagram and document is evaluated then published: the Configuration Management team retrieves these plans and documents, checks the parent-child links to validate the product structure and makes them available to other teams. The Kitting team oversees two documents (Preliminary Equipment List and Kit List) built by using product structure and makes the connection with the Purchase Service. Routing team retrieves the plans made by the Electrical Engineering Office, designs pathways and calculates 
the lengths of cables that will be installed on the aircraft. Its work is also used by the Kitting. Finally, the Wiring Backshop manufactures cables and pre-assembles equipment for later assembly operations in the aircraft, during the production phase.

The whole process is strongly collaborative, nevertheless, there is no collaborative tool for managing all data produced. The Electrical Engineering Office uses a wiring CAD system for design. The Configuration Management team uses a specific product structure manager tool. Validated diagrams from the CAD system are stored into a specific database to be viewed by Purchase Service and Production Department through the company ERP tool $\left(\mathrm{SAP}^{\circledR}\right)$. Other teams use Excel $^{\circledR}$ files to work and to exchange with other teams.

Our challenge is to propose models then tools that will improve collaboration between all actors involved during the whole product life-cycle for the retrofit activity. We propose in the next section a multi-expert knowledge model that will be used to specify the future PLM system.

\section{An integrated model for sharing experts' knowledge}

In this situation of airplane retrofit, a structured and integrated knowledge model must:

- manage product maturity from the definition of a business agreement to the assembly of defined furniture into the airplane;

- manage collaboration, i.e. data exchanges and coherence, and activities' synchronisation between expert actors;

- support knowledge management between different projects to be able to re-use experience;

- support knowledge sharing between different phases of product lifecycle, according to industry 4.0 approach.

In previous work, [10], [8] [11], we proposed to integrate three different dimensions, product, process and organisation/collaboration, to structure an integrated knowledge model, called the PPO model. The objective of integrating such three dimensions is to support the management of the expert know-how during product development process and to enable the efficient coordination of product lifecycle. This knowledge model was studied in a mechanical context but this work had similar objectives with the airplane retrofit case study. 


\subsection{Product modelling}

Product knowledge is a major issue, generally based on a breakdown into several levels of abstraction such as in the FBS model (Function-Behaviour-Structure) proposed by [12]. It must support the possibility for design experts to define specific concepts and to share them in a simple way. But it must support also the management of product maturity in order that other expert actors may use the relevant information for their own activities.

In this specific domain of electrical engineering for aeronautics, the 'product' has not the same meaning as in mechanics. The product is a set of technical elements (wires, connectors, electrical systems, etc. but also assembled or bought systems), linked together. They are defined through several sets of drawings corresponding at different levels of maturity of the design process. For example, an assembled system may be a furniture, containing different bought systems connected with wires, connectors, etc. A bought system can be a power supply system, a routing system, a television or a phone.

We must consider this very specific aspect of electrical design: a technical element cannot be defined on a single drawing. One drawing will define several elements with heir connecting characteristics and an element is defined through several drawings. This set of elements and systems corresponds to a graph structure, and its complexity is very high due to their numerous occurrence: several thousands of items. To control this complexity actors generate different kind of diagrams but the validation is mainly manual for different experts. The knowledge model to be proposed must allow more automated control by integrating knowledge rules from experts.

As we can see in figure 2, product knowledge is then structured into the product dimension by two main concepts that are generic for all experts: the 'document' concept and the 'functional component' concept. As in [13], a PLM system manages product data and related documents even if some PLM systems are document-based systems, and others are clearly product structure-centred. A PLM dedicated to electrical engineering clearly needs to integrate both concepts to facilitate the management of data by the experts. The 'functional component' is always associated to a 'part' which represents its physical instantiation through a unique identification number. As a system, a 'functional component' can be selfdecomposed. It is always 'connected' to other 'functional components'.

The 'Diagram' is the generic document designed or used by all experts, that can be decomposed into himself to detail the solutions, and that is associated to other 'diagrams' to manage the evolution of the solution according to FBS approach. Thus, an implemented diagram can be a layout diagram, then a block diagram, a schematic diagram, and finally a wiring diagram, according to the design progress from first customer specifications analysis to detailed design phase.

As many PLM systems [14], previous concepts are managed using 'lifecycle' concepts to control their maturity level. This is not represented in figure 1 . 

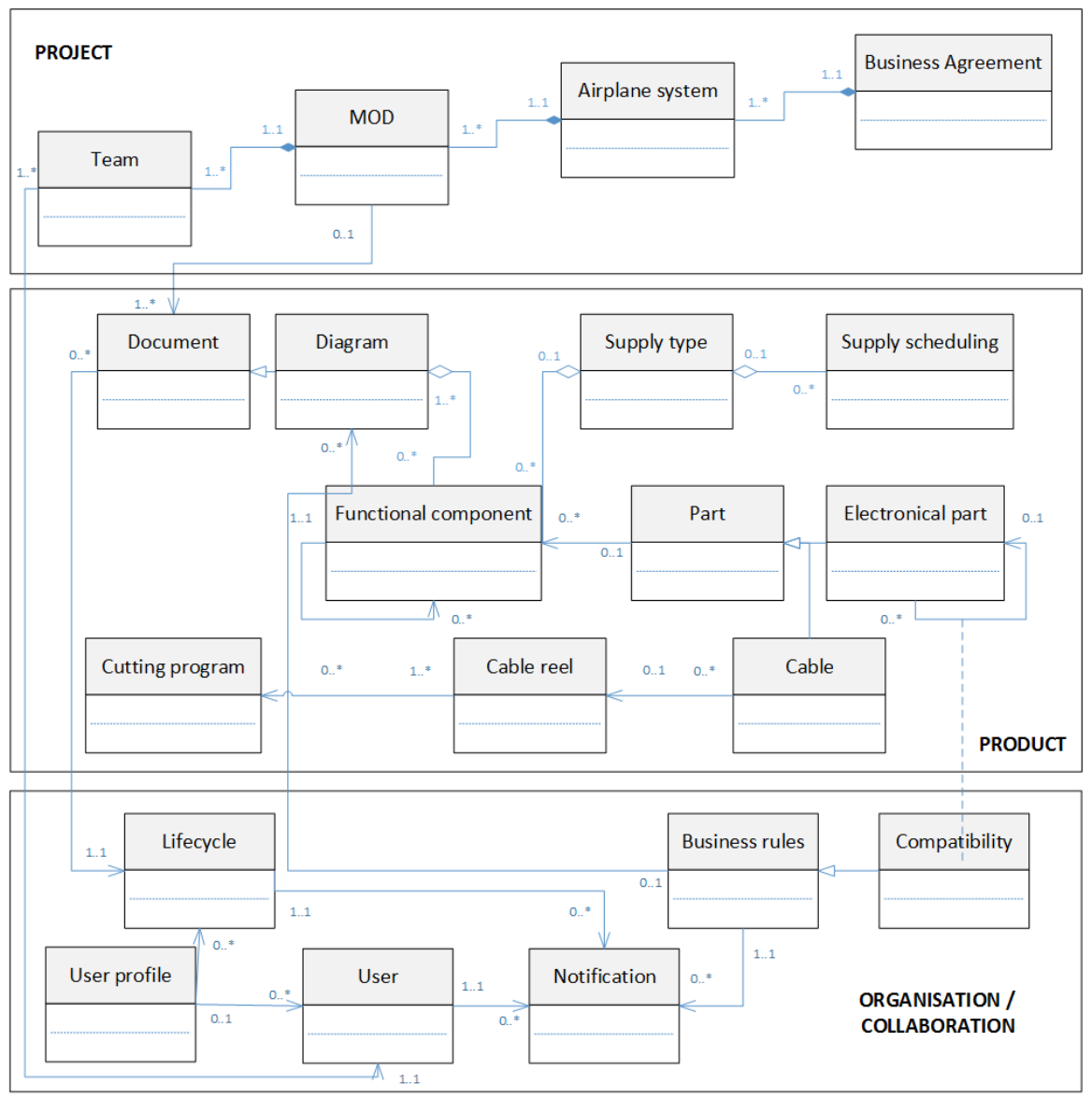

Fig. 2. An integrated knowledge model for electrical engineering in aeronautics

Then several expert concepts have been defined to enrich generic concepts. For example:

- 'Kitting' experts will define how a 'functional component' will be furnished using a 'supply type' concept.

- 'Routing' experts need a 'cable' concept to define the characteristics of the wires (sub-concept of the 'functional component' concept) that have been identified by electrical designers.

- 'Kitting' experts then add the manufacturing characteristics of this specific kind of 'functional component'/'part', then define the 'cable reel' i.e. the initial raw material used for producing the required 'cables'.

- Then 'Wiring backshop' experts generate 'cutting program' which is a specific 'document' that allows to produce all 'cables' from the 'cable reel'.

- These concepts are used again by the 'kitting' experts to prepare the 'cable reel' purchase by defining a 'supply scheduling' concept. 
Such product dimension allows the different experts to achieve their specific business activities by formalising expert knowledge and sharing it throughout few shared concepts.

\subsection{Process and organisation/collaboration modelling}

The process dimension is generally associated to project management concepts by characterising activity sequences [15]. In the case study, and due to the low maturity of the company, we have focused on the project structuring without implementing a formal process dimension. The project is then modelled through the 'business agreement' concept, and is decomposed into 'airplane system' concept which corresponds to a standard decomposition of an airplane into sub-systems impacted by customer specifications. Then each 'airplane system' is divided into 'MOD' concept which corresponds to a set of functional MODifications, according to customer specifications. This decomposition helps managing human resources by allocating experts ('team' concept) to specific 'MODs'. It helps also experts to search information by filtering on a single 'MOD'.

The organisation dimension must allow defining the different stakeholders and their expertise and facilitate collaboration between them all along the product lifecycle, as studied by [16]. Main concept is then 'user profile', which characterizes the different experts, their skills and their access rights to product information according to the phases of the 'product lifecycle', the status of product data and the 'MODs' they have in charge.

The aim of the proposed model is to improve the collective performance of the whole lifecycle. In the product dimension, we already formalise expert knowledge but we only consider knowledge corresponding to information produced by an expert. In order to improve collaboration, we propose now to formalise also rules and methods that can be captured from expert experience and that will automate activities with low added-value. We define an 'expert rule' concept that will formalise links between generic and expert concepts from product, project and organisation dimensions. A 'business rule' is used to detect specific and predefined events such as a change of maturity level of a 'document' and/or a 'functional component' then to start impacting actions to relevant experts. These events are described through a 'notification' action.

For example, when a schematic 'diagram' reaches the state "Released", a specific 'business rule' is activated first. Second the expert/'user' associated to the document is identified: here the 'kitting' user. Third it sends a notification to the 'kitting' user to inform that the document is available. Finally, the 'kitting' user edits the Preliminary Kit List and achieves its activities. This example is the most common way of using 'business rules'.

More generally, the 'business rules' mechanism allows automating the communication flows and the impacts of the solution maturity evolution for the differ- 
ent experts, as already studied in [17]. Some rules require additional concepts to manage more complex links such as in figure 1 an electronic part with a specific 'compatibility' rule.

\section{Towards an electrical engineering PLM system}

\subsection{PLM implementation}

Actually, the PLM system is under development. Both architecture and business oriented Man-Machine Interfaces are defined. Generic and expert concepts are implemented. Automated business rules are implemented through the definition of new functions and they are integrated to existing functions in a transparent way: for example for automated evolution controls, coherency evaluation or impact evaluation. The implemented architecture is described in figure 3 and proposes an access through the CAD system (functions in white boxes) or through a Webbased interface (grey boxes). White-to-grey boxes designate functions that are available on both interfaces.

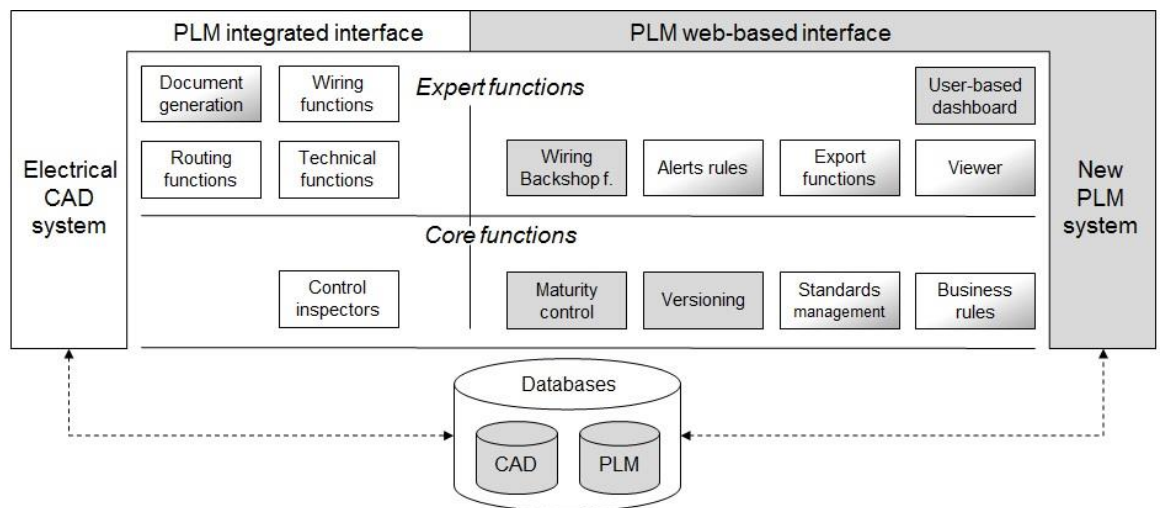

Fig. 3. Architecture of the implemented electrical PLM system

CAD interface is built for electrical design department, wiring and kitting whom tasks require a direct use of electrical diagrams. Web-based interface is dedicated to collaboration and proposes a dashboard customised for each expert profile. Standard viewer function, versioning and maturity management functions are implemented. Expert functions are available, as for example extraction of manufacturing diagrams by 'wiring backshop' experts. Specific standards management (e.g. libraries) and other business rules are also implemented. 
Figure 4 shows the integration of a new PLM function into the CAD system: on the top-right of the figure, four tabs correspond to the four types of diagram. For each tab, the different functions are listed below. Finally, on the bottom-right are indicated tasks depending on the context: here the expert (designer) has the possibility to change the maturity of the schematic diagram from 'work in progress' to 'frozen'. A specific business rule will then send a message to configuration management expert for diagram evaluation.

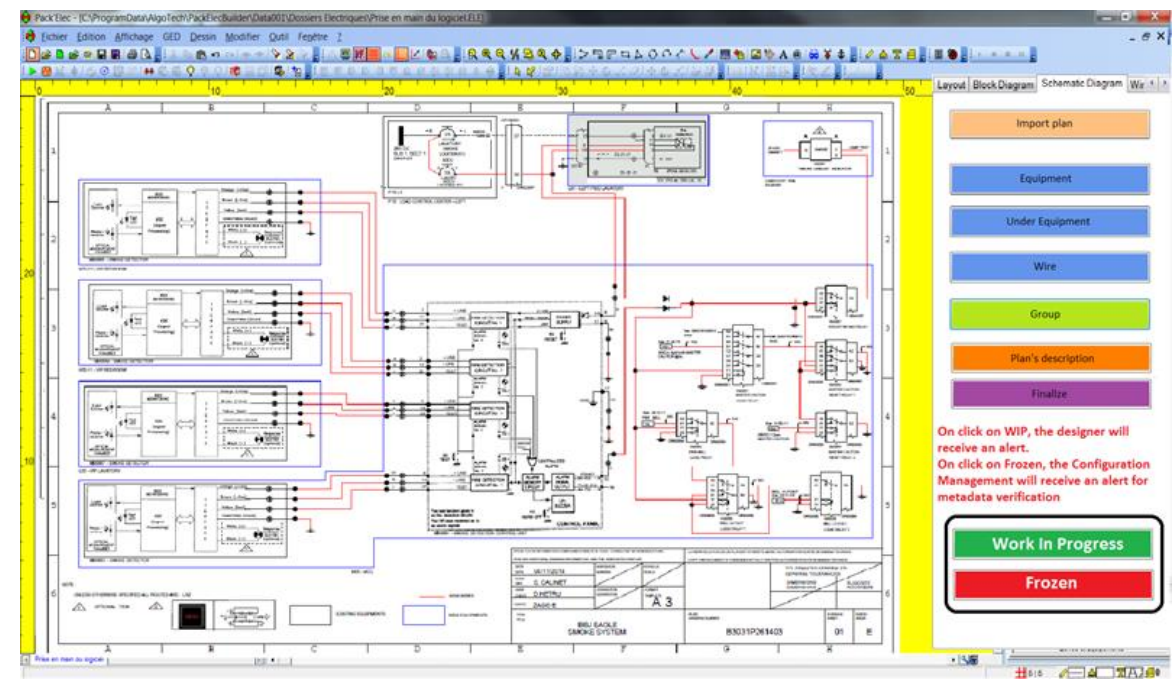

Fig. 4. CAD interface proposal for integrating Diagram generation and evolution

Web-based interface integrates a dashboard of the configuration management expert, where previous message is active. This interface integrates notifications, global overview of projects progress and available functions.

\subsection{Synthesis}

This integrated model provides the core data for developing a new PLM system, coupled with the used electrical CAD system. Product dimension allows managing generic product data and documents, controlling their evolution and the users' access. Expert data extends PLM functions by defining specific business data and associating it to generic product data.

This mechanism supports the definition of automated and parametric 'business rules', defined in the organisation/collaboration dimension. Rules are based on links analysis and cross-referencing between several types of data. Such rules introduce automated communication between experts and flexibility to the system. It 
improves collaboration between experts in a pro-active way by anticipating the expert tasks during the whole development process.

A generic retrofit project generates several thousands of wires and connectors. The coherency of the design, the liability of the solutions and the adequacy with customer specifications were made manually by different experts. Therefore, less errors and reduced development process time are expected with the implementation of the proposed knowledge model.

Nevertheless the complete system still has be evaluated in real situations. Actually, functions integrated to the CAD system have been recently delivered to the company. First results show that all expert rules associated to automated controls generates a qualitative time saving but no quantitative or qualitative study is available. PLM Web interface is still not implemented into the company.

\subsection{Future work}

In this paper we have stated on the first results of our research. Proposing an integrated knowledge model then implementing it through a new PLM system dedicated to electrical engineering builds a strong basis for further research advances.

First work axis relies on the integration of knowledge capitalisation and re-use mechanisms (e.g. experience feedback [18], [19]). One of the most performant methods to implement industrial experience feedback is based on the instrumentation of problem solving methodologies to formalize experiences in the knowledge base [20]. Experience feedback is a useful tool which fits perfectly with the continuous improvement programs implemented in almost all the industrial companies. Indeed, it allows to identify progression tracks by analysing past experiences and to propagate the benefits to similar situations. In a future paper we will propose how project experience can be modelled, implemented and re-used.

We also focused on the product development phases and we do not consider the final phase of installation/production. We have to consider first that the development phases, from the design to the preparation of all components to be assembled, is done without the physical plane. Everything must be ready when the airplane comes to be retrofitted. Operators often discover the real situation of the plane at this moment and modifications are required on the design, and on the furniture. The evaluation of the plane situation by the operators is a long and manual phase that can be improved and time-reduced. Moreover, when operators assemble on the plane the new systems, they spend a lot of time to identify the different locations where the systems, wires, connectors... have to be installed. They can be helped to do these tasks better. Since middle 90', such pre-industrial systems where develop, introducing augmented reality technology on them [21] [22.] [23], but technological environment was not ready. Today it is, and new projects, like SUMATRA project, started in 2016 with same CAD editor, can help improving operators' tasks. The aim of this project is to support industrial maintenance operators in their work around three main principles; having contextual information 
from multiple Information Systems, visualise and manipulate them thanks to modern technologies like augmented reality, and be able to interact with a remote expert thanks to NICT.

Our work will be to adapt such techniques to "mobile terminals" in order to have a new way of interacting and to visualise both the "real view" of the operators, looking at specific elements and systems directly in the plane, and the "virtual view" of the corresponding elements and systems as known by the PLM system. This work will completely integrate the production phase as an operational phase of the product lifecycle management strategy of the company. Moreover, it applies several technologies and methods fostered by Industry 4.0 approach to transform step by step the studied company towards a more digital company.

\section{Conclusion}

In this paper we study how knowledge modelling and computer-supported collaboration participate to the industry 4.0. Our work is based on aeronautical context, for electrical companies. We have proposed an integrated knowledge model composed of three dimensions: product modelling, project modelling and organization/collaboration modelling. We identify generic and expert knowledge and automated mechanisms modelled through 'business rules' in order to support proactive collaboration. This model is actually used by the CAD editor to implement its corresponding PLM systems. We will be involved in the experiments. Further work will concern the generalisation of such model to be applicable in nonaeronautical contexts.

Acknowledgments This work was supported by French Interministerial Unique Funding. We thank all partners from $2 \pi$-MCO project, and especially Jean-Jacques Péré-Laperne, as project leader, Eric Zago, manager of the Electrical Design department, with whom we worked deeply and friendly, and Anthony Cianni who made a great job in our team.

\section{References}

1. Chen, L.: Development of a Multidisciplinary Collaborative Design System. In: Materials Science Forum. 628-629, 31-36 (2009)

2. Bouikni, N., Desrochers, A., Rivest, L.: A Product Feature Evolution Validation Model for Engineering Change Management. Journal of Computing and Information Science in Engineering. 6, 188-196 (2006)

3. Abramovic, M., Bellalouna, F.: Integration and Complexity Management within the Mechatronics Product Development. Advances in Life Cycle Engineering for Sustainable Manufacturing Businesses. 113-118 (2007)

4. Girard, Ph., Robin, V.: Analysis of collaboration for project design management. In: Computers in Industry. 57(8-9), 817-826 (2006) 
5. Nosenzo, V., Tornincasa, S., Bonisoli, E., Brino, M.: Open questions on Product Lifecycle Management (PLM) with CAD /CAE integration. International Journal on Interactive Design and Manufacturing (IJIDeM). 8(2), 91-107, Springer Paris (2014). DOI 10.1007/s12008-0130184-1.

6. Pinquie, R., Rivest, L., Segonds, F., Veron., P.: An illustrated glossary of ambiguous PLM terms used in discrete manufacturing. International Journal of Product Lifecycle Management. 8(2), 142-171, Inderscience (2015)

7. Jones, D., Chanchevrier, N., McMahon, C., Hicks, B.: A Strategy for Artefact-Based Information Navigation in Large Engineering Organisations. In: DS 80-10 Proceedings of the 20th International Conference on Engineering Design (ICED 15). Design Information and Knowledge Management. 10, 143-152, Ed. Milan (2015)

8. Robin, V., Merlo, C., Pol, G., Girard, P.: Management of a Design System in a Collaborative Design Environment Using PEGASE. In: P. Heisig, P.J. Clarkson, S. Vajna (Eds.), Modelling and Management of Engineering Processes, 189-200, Springer London, London (2010)

9. McMahon, C., Lowe, A., Culley, S.: Knowledge management in engineering design: personalization and codification. Journal of Engineering Design. 15(4), 307-325 (2004)

10. Noel, F., Roucoules, L.: The PPO design model with respect to digital enterprise technologies among product life cycle. International Journal of Computer-Integrated Manufacturing, 21(2), 139-145 (2008)

11. Yesilbas, L.G., Rose, B., Lombard, M.: Specification of a repository to support collaborative knowledge exchanges in IPPOP project. Computers in Industry. 57(8-9), 690-710 (2006)

12. Umeda, Y., Takeda, H., Tomiyama, T., Yoshikawa, H.: Function, behaviour and structure. Applications of Artificial Intelligent in Engineering. Springer-Verlag, Berlin (1990)

13. Mickaël, D., Frantz, R.: What does PLMS (product lifecycle management systems) manage: Data or documents? Complementarity and contingency for SMEs. Computers in Industry. 75, 140-150 (2016). ISSN 0166-3615

14. Terzi, S., Bouras, A., Dutta, D., Garetti, M., Kiritsis, D.: Product lifecycle management from its history to its new role. International Journal of Product Lifecycle Management. 4(4), 360-389 (2010)

15. Ullman, D.G.: The mechanical design process. McGraw-Hill, New-York (2009)

16. Belkadi, F., Troussier, N., Eynard, B., Bonjour, E.: Collaboration based on Product Lifecycles Interoperability for Extended Enterprise. International Journal on Interactive Design and Manufacturing. 4(3), 169-179 (2010)

17. Wehbe, A., Merlo, C., Pilnière, V.: Integrating competence management into a coupled project-system design management. In: Advances in Production Management Systems. Competitive Manufacturing for Innovative Products and Services, APMS 2012, Springer, Part III, IFIP AICT 397, 630-637 (2013). ISBN 978-3-642-40351-4

18. Rakoto, H., Hermosillo, J., Ruet, M.: Integration of experience based decision support in industrial processes. In: IEEE International Conference on Systems, Man and Cybernetics. 7 (2002)

19. Villeneuve, E., Béler, C., Pérès, F., Geneste, L.: Hybridization of statistical and cognitive experience feedbacks to per-form risk assessment. In: IEEE International Conference on Industrial Engineering and Engineering Management (IEEM), 10-13, Hong-Kong, China (2012)

20. Bejarano, R., Camilo, J. Coudert, T., Vareilles, E., Geneste, L., Aldanondo, M., Abeille, J.: Case-based reasoning and system design: An integrated approach based on ontology and preference modeling. Artificial Intelligence for Engineering Design, Analysis and Manufacturing. 28(1), 49-69 (2014). ISSN 0890-0604

21. Caudell, T.P., Mizell, D.W.: Augmented reality: an application of heads-up display technology to manual manufacturing processes. In: Proceedings of the Twenty-Fifth Hawaii International Conference on System Sciences, 2, 659-669 (1992)

22. ARVIKA: Augmented reality for development, production, servicing. http://www.arvika.de, (1999)

23. ARMAR: Augmented Reality for Maintenance and Repair. http://graphics.cs.columbia.edu/projects/armar/, (2007) 